\title{
NOGO is increased and binds to BACE1 in sporadic inclusion-body myositis and in A $\beta$ PP-overexpressing cultured human muscle fibers
}

\author{
Slawomir Wojcik $\cdot$ W. King Engel $\cdot$ Riqiang Yan • \\ Janis McFerrin • Valerie Askanas
}

Received: 16 July 2007 / Revised: 6 August 2007 / Accepted: 7 August 2007 / Published online: 31 August 2007

(C) Springer-Verlag 2007

\begin{abstract}
Increased amyloid- $\beta$ precursor protein (A $\beta \mathrm{PP})$ and amyloid- $\beta$ (A $\beta$ ) accumulation appear to be upstream steps in the pathogenesis of sporadic inclusion-body myositis (s-IBM). BACE1, participating in $\mathrm{A} \beta$ production is also increased in s-IBM muscle fibers. Nogo-B and Nogo-A belong to a family of integral membrane reticulons, and Nogo-B binding to BACE1 blocks BACE1 access to A $\beta$ PP, decreasing $A \beta$ production. We studied Nogo-B and Nogo$A$ in s-IBM muscle and in our IBM muscle culture models, based on $\mathrm{A} \beta \mathrm{PP}$-overexpression or ER-stress-induction in cultured human muscle fibers (CHMFs). We report that: (1) in biopsied s-IBM fibers, Nogo-B is increased, accumulates in aggregates, is immuno-co-localized with BACE1, and binds to BACE1; Nogo-A is undetectable. (2) In CHMFs, (a) $\mathrm{A} \beta \mathrm{PP}$ overexpression increases Nogo-B, Nogo-A, and BACE1, (b) ER stress increases BACE1 but decreases Nogo-B and Nogo-A, (c) Nogo-B and Nogo-A associate with BACE1. Accordingly, two novel mechanisms, A $\beta$ PP overexpression and ER stress, are involved in Nogo-B and Nogo-A expression in human muscle. We propose that in
\end{abstract}

Supported by grants (to VA) from the National Institutes of Health (AG 16768 Merit Award), the Muscular Dystrophy Association, and The Myositis Association (to VA), and the Helen Lewis Research Fund.

S. Wojcik · W. K. Engel · J. McFerrin · V. Askanas $(\bowtie)$ USC Neuromuscular Center, Department of Neurology, University of Southern California Keck School of Medicine, Good Samaritan Hospital, 637 S. Lucas Ave,

Los Angeles, CA 90017-1912, USA

e-mail: askanas@usc.edu

R. Yan

Department of Neurosciences, Lerner Research Institute,

The Cleveland Clinic Foundation,

Cleveland, OH 44195, USA
s-IBM muscle the Nogo-B increase may represent an attempt by muscle fiber to decrease $A \beta$ production. However, the increase of Nogo-B seems insufficient because $\mathrm{A} \beta$ continues to accumulate and the disease progresses. We propose that manipulations, which increase Nogo-B in s-IBM muscle might offer a new therapeutic opportunity.

Keywords Inclusion-body myositis $\cdot$ Nogo-B $\cdot$ Nogo-A . Reticulons · Amyloid-beta precursor protein $\cdot$ Amyloid- $\beta$. BACE1

\section{Introduction}

Sporadic inclusion-body myositis (s-IBM) is the most common, relentlessly progressive muscle disease of older persons. It leads to severe muscle wasting and disability, and there is no persistently successful treatment [14]. Both, degeneration of muscle fibers and mononuclear cell inflammation are components of s-IBM muscle biopsy pathology [reviewed in 9, 13]. Degeneration of s-IBM muscle fibers includes vacuolization, accompanied by intra-muscle-fiber accumulations of congophilic, ubiquitinated, multi-protein aggregates, which include amyloid- $\beta(\mathrm{A} \beta)$ and paired helical filaments (PHFs) containing phosphorylated tau (p-tau) $[8,9]$. An intriguing aspect is that the molecular-morphologic phenotype of s-IBM muscle is similar to Alzheimer-disease $(\mathrm{AD})$ brain $[8,9]$ including, in addition to accumulated A $\beta$ and p-tau, accumulations of several other "Alzheimercharacteristic" proteins, markers of oxidative stress, and mitochondrial abnormalities [8, 9]. Endoplasmic reticulum stress and proteasome inhibition were also recently shown to be components of the s-IBM pathogenesis $[15,37]$. Along with accumulated $\mathrm{A} \beta$, there are increased transcription and accumulation of amyloid- $\beta$ precursor protein 
$(\mathrm{A} \beta \mathrm{PP})[3,28]$. BACE1 ( $\beta$-site of the $\mathrm{A} \beta \mathrm{PP}$ cleaving enzyme) [23] and components of $\gamma$-secretase-presenilin 1 and nicastrin [38] —are also increased [7, 34-36] and are known to participate in abnormal processing of $\mathrm{A} \beta \mathrm{PP}$ and $\mathrm{A} \beta$ production. Several lines of evidence based on the IBM human muscle culture model and various transgenic mouse models [16, 20, 21, 31], strongly suggest that intra-musclefiber accumulation of $\mathrm{A} \beta \mathrm{PP}$ and of its proteolytic fragment $\mathrm{A} \beta$ plays a key upstream toxic role in s-IBM pathogenesis $[5,6,8,9,15,24,41,43]$. Accordingly, we postulate that methods reducing $\mathrm{A} \beta$ accumulation in s-IBM muscle fibers could benefit s-IBM patients.

In vitro Nogo-B and RTN3 interact with BACE1 to block BACE1 access to $\mathrm{A} \beta \mathrm{PP}$ resulting in decreased $\mathrm{A} \beta$ production [18]. Nogo, also known as reticulon 4 (RTN4), belongs to the reticulon (RTN) family of integral membrane proteins [32, 44]. All RTNs, including members of the Nogo family, share a common C-terminal domain but differ in the length of their N-terminal domains [32, 44]. Nogo-A (RTN4A), Nogo-B (RTN4B) and Nogo-C (RTN4C) are the three main isoforms of RTN4 [44]. They are encoded by the same gene, and their variants, generated by alternative splicing or alternative promoter usage, are differentially distributed in various tissues [25, 32, 44]. RTNs are especially abundant in the endoplasmic reticulum $[32,44]$. Nogo-B has also been reported in cell surface caveolae/lipid rafts [1]. Nogo-A has been reported to participate in several neurodegenerative disorders [reviewed in $44]$, and recently it was shown accumulated in the $\mathrm{A} \beta$-containing senile plaques of Alzheimer-disease brains [17].

We examined Nogo-B, Nogo-A and RTN3 in s-IBM muscle fibers by immunoblotting and immunomorphologic techniques, and evaluated whether they physically associate with BACE1. To explore the possible pathogenic mechanisms involved in Nogo regulation in s-IBM, we utilized our established experimentally-induced IBM culture human muscle models, based either on overexpression of $\mathrm{A} \beta \mathrm{PP}$ through an adenovirus mediated $\mathrm{A} \beta \mathrm{PP}$ gene transfer, or by induction of ER stress [5, 6, 24, 26, 27].

\section{Materials and methods}

\section{Muscle biopsies}

Studies were performed on diagnostic muscle biopsies obtained with informed consent from 37 patients with the following diagnoses: $14 \mathrm{~s}$-IBM; 4 polymyositis; 2 dermatomyositis; 3 vacuolated non-IBM myopathies (including 2 acid-maltase deficiency patients and 1 vacuolar myopathy of unknown origin); 4 peripheral neuropathies; and 10 normal muscles (no detectable neuromuscular disease). All s-IBM biopsies met the diagnostic criteria detailed in [8].
Immunoblots

Immunoblots of s-IBM and control muscle biopsies were performed as previously described [15, 26, 37, 40]. In brief, $20 \mu \mathrm{m}$-thick frozen muscle sections were collected at $-25^{\circ} \mathrm{C}$ and rapidly homogenized on ice in RIPA buffer and protease inhibitor cocktail (Roche Diagnostic GmBH, Mannheim, Germany). Twenty microgram of total muscle protein were electrophoresed in NuPage 3-8\% Tris-acetate gel and $1 \mathrm{X}$ Tris-acetate SDS running buffer. They were then transferred to a nitrocellulose membrane and incubated with a primary antibody overnight at $4^{\circ} \mathrm{C}$. The following well-characterized antibodies against Nogo were used: (a) rabbit polyclonal antibody R461, recognizing Nogo-A/B on immunoblots [18]; (b) goat polyclonal antibody N-18, also recognizing Nogo-A/B [1, 17]; and (c) rabbit polyclonal antibody $\mathrm{H}-300$, recognizing an epitope exclusively present on the N-terminal of Nogo-A [30]. Further, a rabbit polyclonal antibody R454, recognizing RTN3 [18], was also used in preliminary experiments. BACE1 was studied with a mouse monoclonal antibody, clone 61-3E7 [18], and a rabbit polyclonal antibody. Table 1 lists all the antibodies, their dilutions and sources.

After incubation in a primary antibody, membranes were washed and incubated with an appropriate species-specific secondary antibody conjugated to HRP. The blots were developed using the enhanced chemiluminescence system (Amersham Bioscience, Piscataway, NJ, USA). Protein loading was evaluated by actin bands visualized with a mouse monoclonal anti-actin antibody, diluted 1:2,000 (Santa Cruz Biotechnology, Santa Cruz, CA, USA). Omission of a primary antibody was the control for specificity of the reactions, and these always produced negative result.

The immunoreactivity was quantified by densitometric analysis using the Kodak Gel Logic 440 imaging system (Eastman Kodak Company, Rochester, NY, USA). Intensity of the band of interest was calculated in relation to intensity of the actin band.

\section{Statistical analysis}

The statistical significance was determined by Student's $t$ test. The significance level was set at $P<0.05$. All sets of data are presented as means \pm SEM.

Light-microscopic immunocytochemistry

Immunofluorescence was performed on $10-\mu \mathrm{m}$-thick transverse sections of fresh-frozen muscle biopsies of all the diseases and controls, as described [3, 15, 26, 37, 40], using the same antibodies that were used for the immunoblots. Double-immunofluorescence utilized goat polyclonal antibody against Nogo-A/B in combination with one of the 


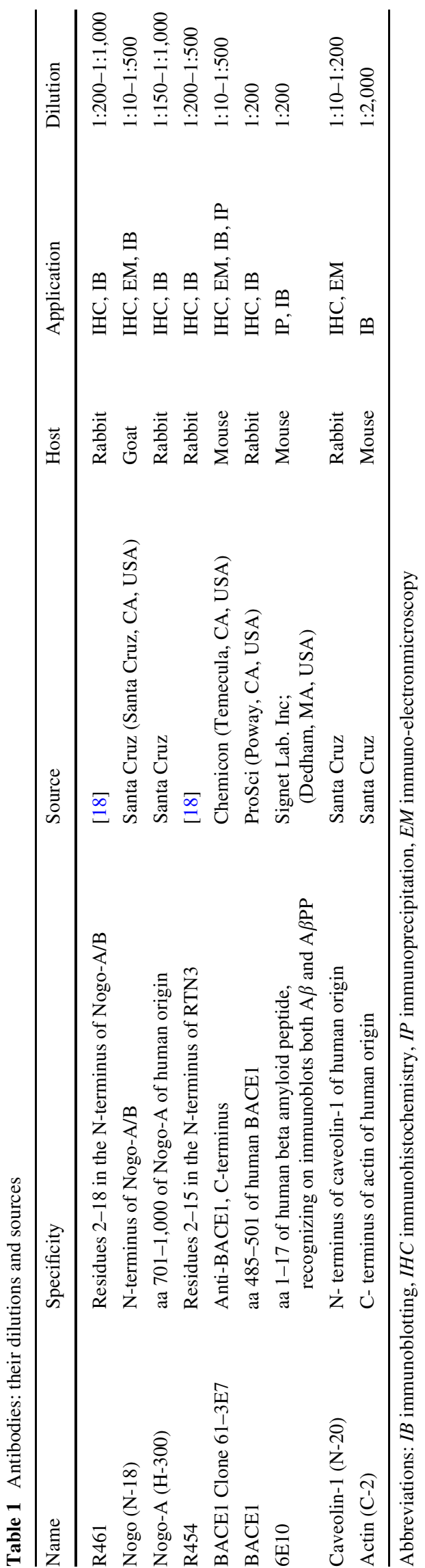

following: (a) mouse monoclonal antibody against BACE1, or (b) rabbit polyclonal antibody against caveolin-1. To block non-specific binding of an antibody to Fc receptors, all sections were preincubated with $10 \%$ normal goat or donkey serum $[15,26,37,40]$. Omission of the primary antibody or its replacement with non-immune sera or irrelevant antibody was the control for staining specificity, and these always produced negative results.

\section{Gold-immuno-electronmicroscopy}

Single- and double-labeled gold immuno-electron-microscopy were performed on 10- $\mu \mathrm{m}$ unfixed frozen sections of s-IBM biopsies adhered to the bottom of 35-mm Petri dishes, as described [3, 15, 26, 37, 40]. Two different species-specific secondary antibodies, conjugated to either 12 or $6 \mathrm{~nm}$ gold particles (Jackson ImmunoResearch Laboratories, West Grove, PA, USA), were used in this study. The sections were then processed for EM as previously described $[3,15,26,37,40]$.

\section{Combined immunoprecipitation-immunoblotting}

To evaluate whether in s-IBM muscle fibers (a) Nogo physically associates with BACE1, and (b) BACE1 physically associates with $\mathrm{A} \beta \mathrm{PP}$, we performed the combined immunoprecipitation-immunoblot technique, as described $[15,37,40,41]$. In brief, $100 \mu \mathrm{g}$ of total muscle protein were immunoprecipitated in precipitation-buffer containing $5 \mu \mathrm{g}$ of IgG antibody against BACE1. The immunoprecipitated complex, containing $\operatorname{IgG}$ antibody along with its bound target antigen and all proteins bound to that antigen, was pulled down using Protein G Sepharose 4 Fast Flow (Amersham) during $4 \mathrm{~h}$ of incubation at $4^{\circ} \mathrm{C}$. The solution was then centrifuged for $5 \min \left(16,000 \times g\right.$ at $\left.4^{\circ} \mathrm{C}\right)$ and the supernatant removed. The precipitated sepharose immunocomplexes were washed three times with the precipitation buffer by centrifuging $5 \mathrm{~min}$ each $\left(16,000 \times g\right.$ at $\left.4^{\circ} \mathrm{C}\right)$. Immunoprecipitates were electrophoresed and immunoprobed with either goat polyclonal anti-Nogo antibody or mouse monoclonal $6 \mathrm{E} 10$ antibody against $\mathrm{A} \beta \mathrm{PP} / \mathrm{A} \beta$, each followed by an appropriate secondary antibody. To confirm specificity of the physical association identified by the immunoprecipitation-immunoblot reaction, primary antibodies were omitted from either the immunoprecipitation or immunoprobing. The same technique was performed to evaluate whether Nogo physically associates with A $\beta$ PP.

\section{Cultured human muscle fibers}

Primary cultures of normal human muscle were established, as we have described [2], from satellite cells of portions of diagnostic muscle biopsies from patients who, after all tests 
were performed, were considered free of muscle disease. We established 11 culture sets, each from satellite cells derived from a different muscle biopsy. All experimental and control conditions were studied on sister cultures in the same culture set. Not all studies were performed on every set. Twenty days after myoblasts fused, a $3 \mathrm{~Kb} 751$ A $\beta$ PP-cDNA, in either sense or anti-sense orientation, was transferred into well-differentiated myotubes using a replication-deficient adenovirus (RDAV) vector at $0.3 \times$ $10^{8} \mathrm{pfu} / \mathrm{ml}$ culture medium, as detailed $[5,6,24]$. At the same time, non-A $\beta$ PP-transduced control sister-cultures were treated for $24 \mathrm{~h}$ with either of two ER-stress inducers, Tunicamycin, an $N$-glycosylation inhibitor $(4 \mu \mathrm{g} / \mathrm{ml})$, or Thapsigargin, an inhibitor of ER calcium-ATPase (300 nM) (both inhibitors from Sigma Co, St Louis, MO, USA) $[10,22,26]$. In our previous studies, both inhibitors successfully induced ER stress in CHMFs [26, 27]. Four days after $\mathrm{A} \beta \mathrm{PP}$ gene transfer, or $24 \mathrm{~h}$ after treatment with an ER-stress inducer, cultures were processed for light-microscopic immunocytochemistry, immunoblot and immunoprecipitation studies of Nogo and BACE1, as described [15, 26, 41, 43].

\section{Results}

Muscle biopsies

Nogo-B and BACE1 are increased in S-IBM muscle fibers

Antibodies R461 and N-18, which were previously characterized as recognizing both Nogo-A and Nogo-B [1, 17, 18], showed a strongly increased level of $46 \mathrm{kDa}$ Nogo-B in s-IBM as compared to controls (Fig. 1a). Densitometric quantification of immunoblots performed on $7 \mathrm{~s}-\mathrm{IBM}$ and 7 control muscle biopsies revealed that Nogo-B was increased 2.5-fold $(P=0.025)$ in s-IBM biopsies compared to controls (Fig. 1b). Those antibodies R461 and N-18 should also recognize Nogo-A; however, Nogo-A was not expressed in either normal-control or s-IBM muscle biopsies (Fig. 1a). (In our studies, similarly to previous reports $[17,30]$, Nogo-A has molecular weight of approximately $180 \mathrm{kDa}$, which may be due to Nogo-A glycosylation.) Another Nogo-A specific antibody, H-300 (Santa Cruz) also failed to detect any Nogo-A in these samples; a weak band corresponding to $80 \mathrm{kDa}$ was non-specific (Fig. 1a). We found that all three of these antibodies did recognize Nogo-A in a control lysate from human medulloblastoma cells (TE671) that express Nogo-A (Fig. 1a), and also in muscle biopsies from patients with peripheral neuropathy and ALS [42, not shown here]. Together, our results showed that Nogo-B levels are significantly increased in s-IBM while Nogo-A is not detectable. The level of RTN3, another important inhibitor of BACE1 [18], was low in both s-IBM and control biopsies, and there was no discernable difference between the two groups (data not shown). BACE1, which we have previously shown increased and migrating at $70 \mathrm{kDa}$ in s-IBM muscle fibers [34, 36], was, when quantified in the current studies, found increased 3.5 -fold $(P=0.007)$ in s-IBM as compared to control muscle biopsies (Fig. 1c, d).

\section{Detection of protein aggregates containing Nogo-B and BACEI in s-IBM}

We used immunohistochemistry to morphologically localize Nogo-B in s-IBM muscle fibers. In all of the s-IBM muscle biopsies, $60-70 \%$ of the vacuolated muscle fibers contained, mainly in their non-vacuolated cytoplasm, numerous welldefined, plaque-like, punctuate or elongated, aggregates immunoreactive with antibodies R461 and N-18 (Fig. 2a, c, d, f). As shown above by our immunoblots, these two antibodies in s-IBM muscle recognize exclusively Nogo-B, suggesting that the morphologically evident aggregates contain Nogo-B. In addition, in all s-IBM muscle biopsies, 20-30\% of the non-vacuolated fibers (on a given cross-section) contained similar aggregates (Fig. 2b). Nogo-B immunoreactive aggregates also contained BACE1 (Fig. 2d, e). As both BACE1 and Nogo-B can be localized in lipid rafts, we found with an antibody against caveolin-1, that caveolin-1 was associated with some of the Nogo-B aggregates (Fig. 2f, g). These results appeared specific because the antibody H-300, which specifically recognizes Nogo-A, and did not detect any specific band in s-IBM muscle biopsies on immunoblots, did not label any aggregates. In contrast to the staining of Nogo-B in s-IBM biopsies, none of the normal or disease-control biopsies had muscle fibers containing Nogo$\mathrm{B}$ immunoreactive aggregates (data not shown). While Nogo-A was negative in s-IBM muscle fibers, it was, as we have recently reported [42], strongly and diffusely immunoreactive in (a) regenerating muscle fibers in dermatomyositis and polymyositis, and (b) in denervated muscle fibers in amyotrophic lateral sclerosis and peripheral neuropathies (not shown).

\section{Nogo-B and BACE1 ultrastructurally co-localize within s-IBM aggregates}

To determine the ultrastructural localization of Nogo-B in the aggregates, we performed immuno-electronmicroscopic examination of s-IBM muscle fibers. Nogo-B was detected on or very close to the $6-10 \mathrm{~nm}$ amyloid-like fibrils (Fig. 3a), but was more associated with the cytoplasmic amorphous material (Fig. 3b). Double-labeling experiments showed that BACE1 was co-localized with Nogo-B on 6-10 nm filaments and on the amorphous material 


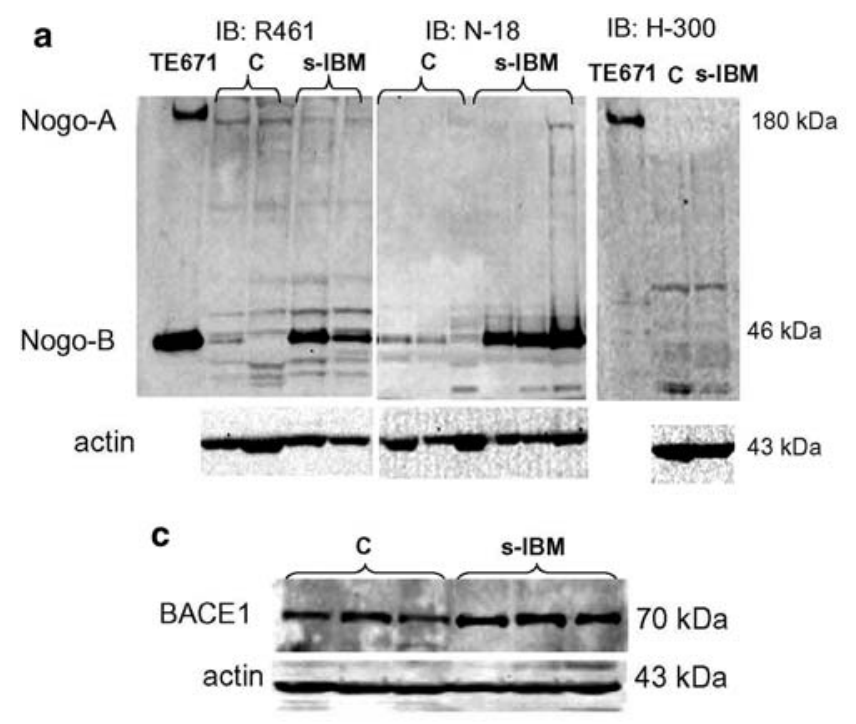

b

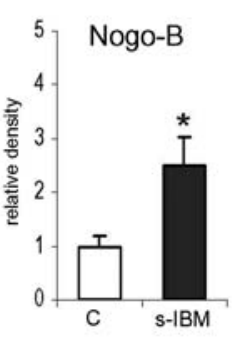

Fig. 1 Immunoblots of Nogo-B, Nogo-A and BACE1 of muscle homogenates of control $(C)$ and s-IBM muscle biopsies. a Demonstrates that a $46 \mathrm{kDa}$ band of Nogo-B detected with antibodies R461 and N-18 was significantly stronger in s-IBM than in control biopsies; however a $180 \mathrm{kDa}$ band corresponding to Nogo-A was not demonstrable with antibodies R461 and N-18, which is considered to recognize both Nogo-A and -B. (This was confirmed with an antibody H-300, which specifically recognizes Nogo-A. Nogo-A was not demonstrable in either control or s-IBM muscle biopsies with this antibody). TE671 indicates immunoblots of an extract of human medulloblastoma cells that express both Nogo-A and -B. In b immunoreactivity was quantified by densitometric analysis using the Kodak Gel Logic 440 imaging system (Eastman Kodak Company, Rochester, NY, USA). Intensity of the band of interest was calculated in relation to the actin band Immunoblot in $\mathbf{c}$ and its densitometric analysis in $\mathbf{d}$ demonstrate that BACE1 is significantly increased in s-IBM as compared to controls. (Details for a-d in the text, * indicates $P<0.05$ )
Fig. 2 Immunofluorescence of Nogo-B in s-IBM muscle fibers. a-c Single-label immunofluorescence illustrates stronglyimmunoreactive, various-sized aggregates of Nogo-B. d-g Double-label immunofluorescence-the aggregates immunoreactive for Nogo-B (d) are also immunoreactive for BACE1 (e), and aggregates immunoreactive for Nogo-B (f) are also immunoreactive for caveolin-1 (g). a-c $\times 1,100$, d-g $\times 2,000$
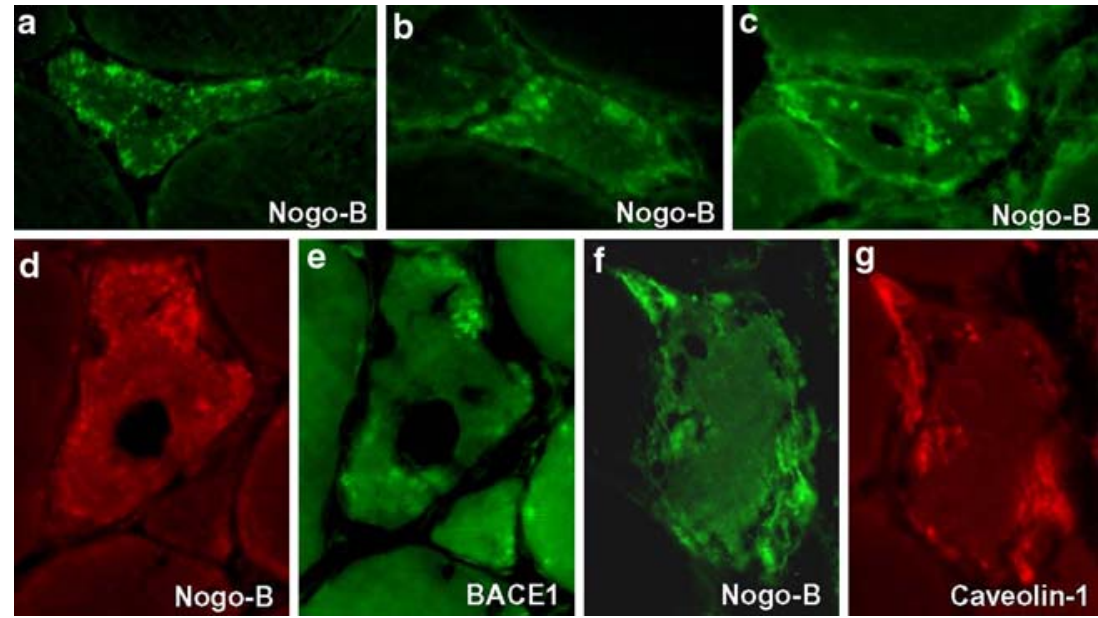

(Fig. 3c). Both Nogo-B and BACE1 were also found in the lipid raft structures that were labeled by the antibody against caveolin-1 (Fig. 3d,e). Therefore, Nogo-B immunoreactive aggregates appear to contain BACE1.

\section{Physical interaction between BACE1 and Nogo-B}

Since the ultrastructural results suggest that BACE1 and Nogo-B co-exist in s-IBM aggregates, we next tested whether, within s-IBM muscle fibers, they physically interact as previously reported in the human brain [18]. Immunoprecipitation with an antibody against BACE1 followed by immunoblotting with antibody $\mathrm{N}-18$, which in s-IBM muscle biopsies recognized only Nogo-B, revealed a $46-\mathrm{kDa}$ band corresponding to Nogo-B. Omission of a primary antibody against BACE1 from the immunoprecipitation reaction (denoted by $x$ ) or in the immunoblot (denoted by \#) failed to detect Nogo-B (Fig. 4a). The co-immunoprecipitation 


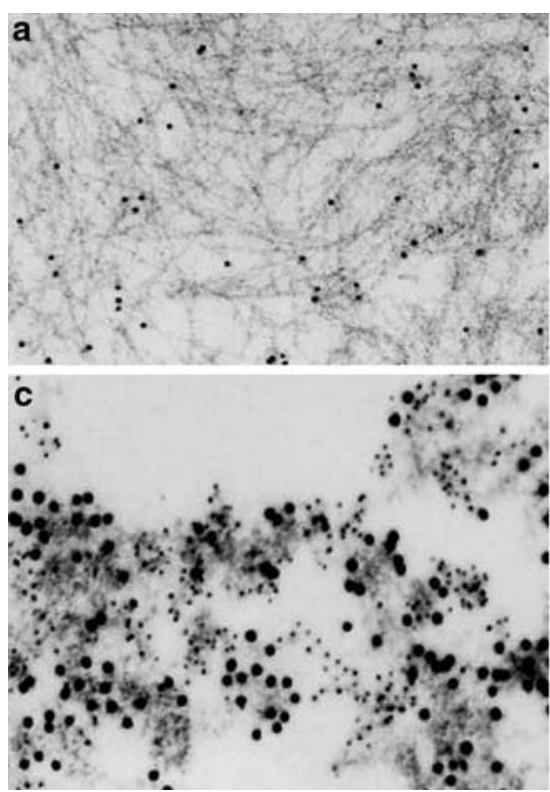

Fig. 3 Single (a, b) and double-label (c, d, e) gold-immuno-electronmicroscopy of s-IBM muscle fibers. a, b Nogo-B (12 nm gold particles) is localized on 6-10 nm filaments (a) and a large amorphous inclusion (b); on the right of $\mathbf{b}$ is a normal myofibrillar structure that does not contain immunoreactive Nogo-B. c Nogo-B (12 nm gold particles) and BACE1 (6 nm gold particles) are associated with $6-10 \mathrm{~nm}$

experiments also demonstrated that in s-IBM muscle biopsies BACE1 interacted with A $\beta$ PP (Fig. 4b). However, there was no physical association of Nogo-B with $\mathrm{A} \beta \mathrm{PP} /$ A $\beta$ (Fig. 4c). Together, these studies indicate that Nogo-B and BACE1 are present in aggregates where they appear to have interacted.

Cultured human muscle fibers

\section{Increased expression of $A \beta P P$ enhances Nogo- $B$ and BACE1 protein levels}

To determine what might be causing increased expression of Nogo-B and BACE1 in s-IBM muscle fibers, we utilized

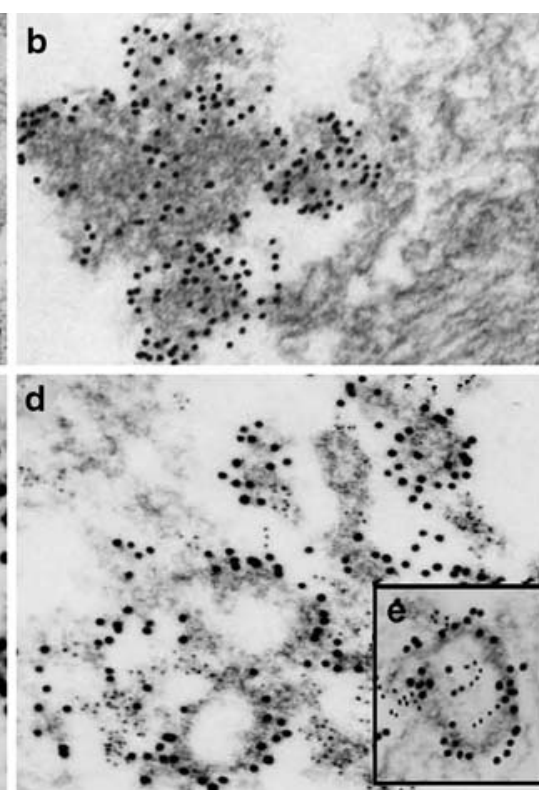

amyloid-like fibrils and with amorphous-floccular material. d both Nogo-B (12 nm gold particles) and BACE1 (6 nm gold particles) are immunolocalized in the caveolae structures, and on 6-10 nm filaments. e Double-labeling of a caveola with antibodies against Nogo-B (12 nm gold particles) and caveolin-1 (6 nm gold particles). a-d $\times 53,000$; c $\times 68,000$

our IBM human culture model, which exhibits several abnormalities present in s-IBM muscle fibers $[6,15,24,41$, 43]. Since increased production and accumulation of $A \beta P P$ and $\mathrm{A} \beta$, as well as ER-stress, seem to be important components of the s-IBM pathogenesis, in this study we: (a) engineered cultured human muscle fibers (CHMFs) to overexpress $\mathrm{A} \beta \mathrm{PP}$ through an adenoviral $\mathrm{A} \beta \mathrm{PP}$ cDNA transfer, and (b) separately induced ER-stress in CHMFs.

Densitometric analysis of immunoblots demonstrated that in the $\mathrm{A} \beta \mathrm{PP}$-overexpressing $\mathrm{CHMFs}$ (lane $\mathrm{A} \beta \mathrm{PP}+$ ) compared to controls (Fig. 5a, b) a $46 \mathrm{kDa}$ band corresponding to Nogo-B was increased 1.3 -fold $(P=0.018)$ and Nogo-A was increased 1.7 fold $(P=0.038)$. A $\beta$ PP overexpression in CHMFs also caused 1.9 -fold $(P=0.022)$

Immunoprecipitation-immunoblotting

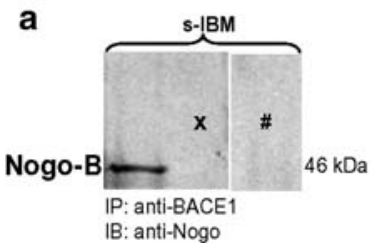

Fig. 4 a, b Immunoprecipitations of s-IBM biopsies with anti-BACE1 antibody, followed by immunoprobing with an antibody against Nogo $\mathrm{A} / \mathrm{B}$ in $\mathbf{a}$, and with antibody $6 \mathrm{E} 10$ against $\mathrm{A} \beta \mathrm{PP} / \mathrm{A} \beta$ in $\mathbf{b}$. A strong band of the appropriate molecular weight of $46 \mathrm{kDa}$ corresponding to Nogo$\mathrm{B}$ is visible in $\mathbf{a}$. In $\mathbf{b}$, a strong band of $130 \mathrm{kDa}$ corresponds to A $\beta \mathrm{PP}$. $X=$ omission of the antibody against BACE1 from the immunoprecip-
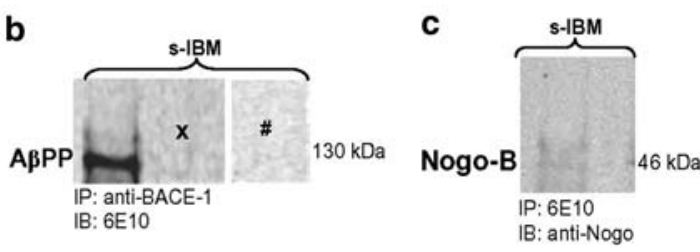

itation solution; \# = omission of the antibody against Nogo-B in a or omission of the antibody against $\mathrm{A} \beta \mathrm{PP} / \mathrm{A} \beta(6 \mathrm{E} 10)$ in $\mathbf{b}$ in the immunoblotting - these omissions resulted in negative reactions. Together the data indicate there is a physical interaction between Nogo-B and BACE1 in s-IBM muscle fibers. $\mathbf{c}$ Illustrates that there is no interaction between $\mathrm{A} \beta \mathrm{PP} / \mathrm{A} \beta$ and Nogo-B 
Fig. 5 Immunoblots performed with cultured human muscle fibers $(C H M F s)$. a Immunoblots of control- $\mathrm{C}$, Tg- and Tmexposed, and $\mathrm{A} \beta \mathrm{PP}$-overexpressing CHMFs, and their

densitometric analyses (b), show that Nogo-A and -B are significantly increased in $\mathrm{A} \beta \mathrm{PP}$-overexpressing CHMFs, but both are decreased in CHMFs in which ER-stress was induced by $\mathrm{Tg}$ or Tm treatment. c, d BACE1 is significantly increased due to A $\beta$ PP overexpression, or treatment with Tg or Tm. e-h Illustrates that overexpressed antisense- $\mathrm{A} \beta \mathrm{PP}$ does not influence Nogo-A or-B, or BACE1 in CHMFs. a-h $*$ indicates $P<0.05$ )
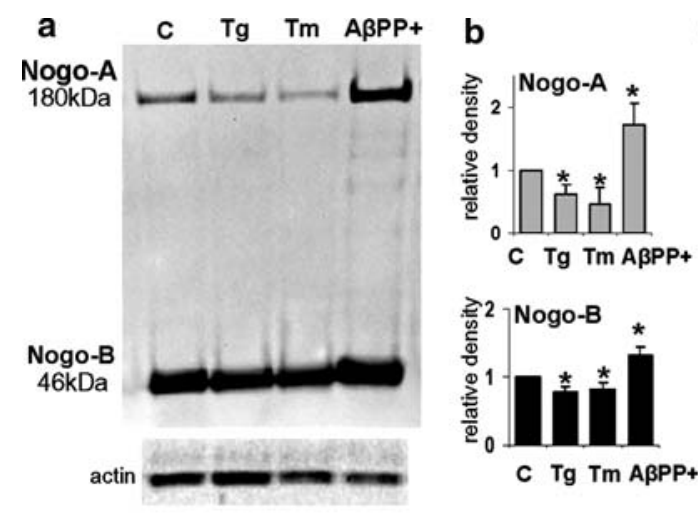

C

\section{d}
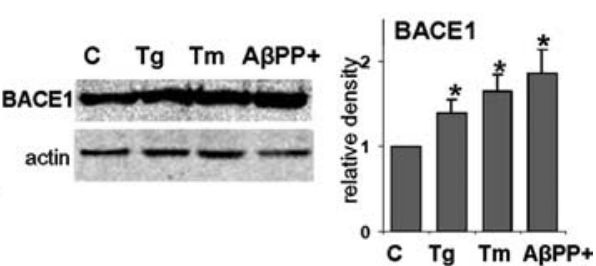
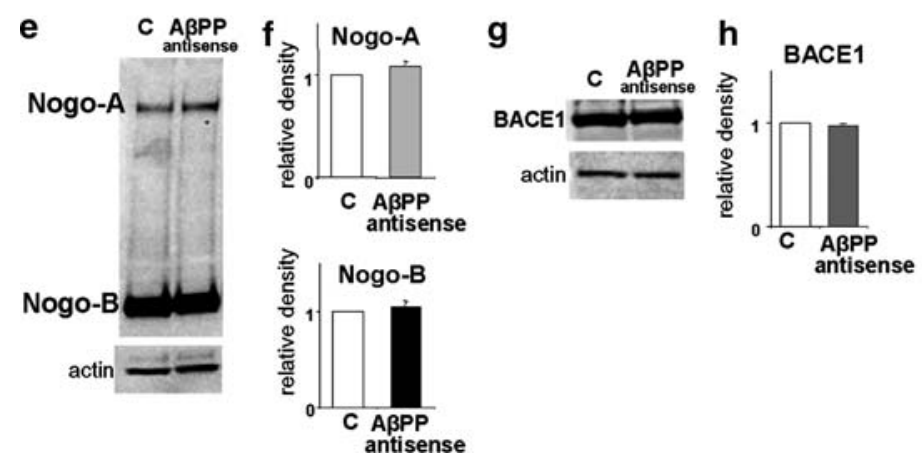

increase of BACE1 as compared to controls (Fig. 5c, d). These effects of $\mathrm{A} \beta \mathrm{PP}$ overexpression were specific because overexpressing $\mathrm{A} \beta \mathrm{PP}$ in antisense orientation in CHMFs did not cause increase of Nogo-B, Nogo-A, or BACE1 (Fig. 5e-h).

Contrarily to $\mathrm{A} \beta \mathrm{PP}$-overexpression, treatment of CHMFs with two ER-stress inducers, thapsigargin ( $\mathrm{Tg}$ ) or tunicamycin $(\mathrm{Tm})$, led to decreased level of Nogo-B (1.3fold $[P=0.019]$ by thapsigargin, and 1.2-fold $[P=0.04]$ by tunicamycin), and of Nogo-A (1.4-fold $[P=0.001]$ by thapsigargin and 1.5 -fold $[P=0.001]$ by tunicamycin) (Fig. 5a, b). However, both ER-stress inducers increased BACE1 levels-1.4-fold $(P=0.027)$ after thapsigargin treatment and 1.7-fold $(P=0.011)$ after tunicamycin treatment (Fig. 5c, d).

By immunofluorescence, in normal, non-A $\beta$ PP-overexpressing CHMFs, Nogo and BACE1 were weakly and

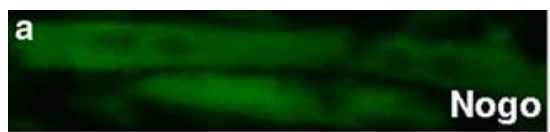

diffusely immunoreactive (Fig. 6a, b), whereas in the $\mathrm{A} \beta \mathrm{PP}-$ overexpressing $(\mathrm{A} \beta \mathrm{PP}+)$ cultures their immunoreactivities were stronger and occasionally in aggregates (Fig. 6c, d). (Because CHMFs express both Nogo-B and Nogo-A, and the antibodies N-18 and R461 that we utilized for immunostaining recognize both Nogo-A and Nogo-B, we use term "Nogo" to refer to those two stainings.) In the aggregates, Nogo and BACE1 co-localized (Fig. 6c, d). In the CHMFs, interactions of BACE1 with Nogo-B and Nogo-A (Fig. 7a) or with A $\beta$ PP (Fig. 7b) were further confirmed through our co-immunoprecipitation experiments.

\section{Discussion}

In s-IBM muscle fibers, we demonstrated by immunoblot analysis, using well-characterized anti-Nogo antibodies,

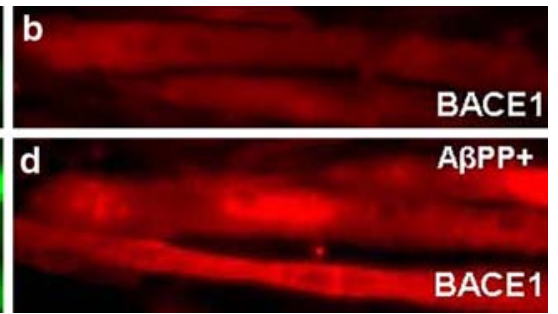

Fig. 6 a-d Double-label immunofluorescence of "Nogo" (a, c) with BACE1 $(\mathbf{b}, \mathbf{d})$, in the control $(\mathbf{a}, \mathbf{b})$ and the A $\beta$ PP-overexpressing $(\mathbf{c}, \mathbf{d})$ CHMFs. This illustrates the co-localization of Nogo and BACE1, and

the stronger immunostaining of both due to the $\mathrm{A} \beta \mathrm{PP}$ overexpression. An upper fiber in $\mathbf{c}$ and $\mathbf{d}$ contains two large aggregates containing both Nogo and BACE1. All $\times 1,200$ 


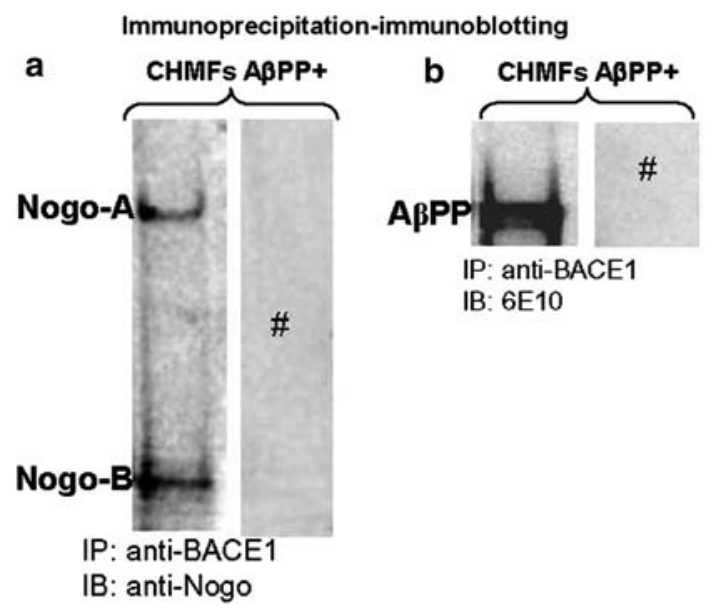

Fig. 7 Immunoprecipitation-immunoblotting illustrates that both Nogo-B and -A associate with BACE1 (a), and BACE1 associates with $\mathrm{A} \beta \mathrm{PP} / \mathrm{A} \beta(\mathbf{c}) . \#=$ omission of the primary antibody from the immunoblotting reaction

that Nogo-B is significantly increased and Nogo-A is virtually undetectable. Our immunocytochemical studies showed that in s-IBM muscle fibers Nogo-B was abnormally accumulated multifocally in the form of aggregates that also contained BACE1 and caveolin-1. By immunoelectronmicroscopy, both Nogo-B and BACE1 were associated with the same ultrastructural components, namely, amorphous material, 6-10 $\mathrm{nm}$ amyloid-like fibrils and caveolae/lipid-rafts-like structures, which also contained caveolin-1. Previous studies in other cells have also shown that Nogo-B and BACE1 are present in the caveolae/lipidrafts structures, and processing of $\mathrm{A} \beta \mathrm{PP}$ leading to $\mathrm{A} \beta$ generation is considered to occur in those structures as well $[1,12,23,33]$.

The two-reticulon family members, RTN3 and Nogo$\mathrm{B}$, were previously shown to bind BACE1 in human brain and in HEK-293 cells [18]. This binding was recently shown to occur through the C-terminal domain common to all reticulons and the C-terminal domain of BACE1 [19]. This binding inhibits association of BACE1 with $\mathrm{A} \beta \mathrm{PP}$ and decreases generation of $\mathrm{A} \beta-40$ and $\mathrm{A} \beta-42$ $[18,19]$. Accordingly, within s-IBM muscle fibers, the increased Nogo-B and its association with BACE1, might reflect an attempt by those fibers to decrease $\mathrm{A} \beta$ production caused by BACE1-mediated abnormal processing of $\mathrm{A} \beta \mathrm{PP}$.

In our cultured human muscle fibers, $\mathrm{A} \beta \mathrm{PP}$ overexpression increased both Nogo-B and Nogo-A, as well as the intracellular amount of BACE1. We propose that the increased BACE1 is in response to an increased demand to process the excessive $\mathrm{A} \beta \mathrm{PP}$, resulting in increased $\mathrm{A} \beta$ production. Although the mechanism(s) of increased Nogo-B and $-\mathrm{A}$, in this system needs to be clarified, we suggest that they might be to inhibit (at least partially) BACE1 binding to $\mathrm{A} \beta \mathrm{PP}$, thereby decreasing production of $\mathrm{A} \beta$ and protecting the muscle fiber from the $\mathrm{A} \beta$ detrimental influence. Since both Nogo-B and Nogo-A bind to BACE1 in our CHMFs, we propose that both Nogo isoforms could protect a muscle fiber from an abnormal accumulation of $\mathrm{A} \beta$; however, in the s-IBM biopsied muscle fibers, only Nogo$\mathrm{B}$ seems to be playing this putative role. Because Nogo-A is increased in regenerating muscle fibers [42], it may have additional roles in those young fibers. One possibility might be to help manage the increased $\mathrm{A} \beta \mathrm{PP}$ known to occur in regenerating muscle fibers in vivo [4, 29] — this putative benefit of Nogo-A could be deficient in s-IBM muscle fibers, since they have diminished regenerative capabilities, and we could not demonstrate Nogo-A in them. Nogo-A is predominantly expressed in the central nervous system, where it is present mainly in oligodendrocytes [11, 39]. A recent study demonstrated that Nogo-A, but not Nogo-B, is increased in hippocampal neurons in Alzheimer disease (AD) brain, and it also co-localizes with $\mathrm{A} \beta$ deposits in extracellular senile plaques [17]. Similarly to our present studies, Nogo-A did not physically associated with $\mathrm{A} \beta \mathrm{PP} / \mathrm{A} \beta$ in $\mathrm{AD}$ brain [17]; whether Nogo- $\mathrm{A}$ physically associated with BACE1 in AD brain was not reported [17].

In our cultured human muscle fibers, ER stress decreased Nogo-A/B and increased BACE1 levels as compared to the unstressed control. Analogously, we suggest that in s-IBM muscle fibers, continuous ER stress [27, 37] might be reducing the intracellular ratio of Nogo-B to BACE1 by decreasing Nogo-B and increasing BACE1, resulting in $\mathrm{A} \beta$ continuing to accumulate and exert its cytotoxic influence.

\section{Conclusions}

We report abnormally increased Nogo-B and its multi-focal accumulation within s-IBM muscle fibers. We experimentally demonstrate that two different mechanisms, namely $\mathrm{A} \beta \mathrm{PP}$ overexpression and endoplasmic reticulum stress, are differentially involved in regulation of Nogo-B, Nogo-A and BACE1 in cultured human muscle fibers. Since both A $\beta$ PP overexpression and ER-stress are important pathologic aspects of the s-IBM muscle fibers, the same regulatory mechanism may operate in them. If so, manipulations toward increasing Nogo-B and/or interfering with pathways inducing ER-stress might provide new therapeutic opportunities for s-IBM patients.

Acknowledgments Slawomir Wojcik is on leave from the Department of Anatomy and Neurobiology, Medical University of Gdansk, Gdansk, Poland. Maggie Baburyan provided excellent technical assistance in electronmicroscopy. 


\section{References}

1. Acevedo L, Yu J, Erdjument-Bromage H, Miao RQ, Kim JE, Fulton D, Tempst P, Strittmatter SM, Sessa WC (2004) A new role for Nogo as a regulator of vascular remodeling. Nat Med 10:382-388

2. Askanas V, Engel WK (1992) Cultured normal and genetically abnormal human muscle, in the handbook of clinical neurology. In: Rowland LP, Di Mauro S (eds) Myopathies, vol 18. North Holland, Amsterdam, pp 85-116

3. Askanas V, Alvarez RB, Engel WK (1993) $\beta$-amyloid precursor epitopes in muscle fibers of inclusion body myositis. Ann Neurol 34:551-560

4. Askanas V, Sarkozi E, Bilak M, Engel WK (1994) Expression of $\beta$-amyloid precursor protein, prion and acetylcholine receptor and their mRNAs in human muscle fibers regenerating in vivo. Brain Pathol 4:322

5. Askanas V, McFerrin J, Baque S, Alvarez RB, Sarkozi E, Engel WK (1996) Transfer of beta-amyloid precursor protein gene using adenovirus vector causes mitochondrial abnormalities in cultured normal human muscle. Proc Natl Acad Sci USA 93:1314-1319

6. Askanas V, McFerrin J, Alvarez RB, Baque S, Engel WK (1997) $\beta$ APP gene transfer into cultured human muscle induces inclusionbody myositis aspects. Neuroreport 8:2155-2158

7. Askanas V, Engel WK, Yang CC, Alvarez RB, Lee VM, Wisniewski T (1998) Light and electron microscopic immunolocalization of presenilin 1 in abnormal muscle fibers of patients with sporadic inclusion-body myositis and autosomal-recessive inclusion-body myopathy. Am J Pathol 152:889-895

8. Askanas V, Engel WK (2001) Inclusion-body myositis: newest concepts of pathogenesis and relation to aging and Alzheimer disease. J Neuropathol Exp Neurol 60:1-14

9. Askanas V, Engel WK (2006) Inclusion-body myositis: a myodegenerative conformational disorder associated with $\mathrm{A} \beta$, protein misfolding, and proteasome inhibition. Neurology 66:S39-S48

10. Back SH, Schroder M, Lee K, Zhang K, Kaufman RJ (2005) ER stress signaling by regulated splicing: IRE1/HAC1/XBP1. Methods 35:395-416

11. Buss A, Sellhaus B, Wolmsley A, Noth J, Schwab ME, Brook GA (2005) Expression pattern of NOGO-A protein in the human nervous system. Acta Neuropathol (Berl) 110:113-119

12. Cordy JM, Hooper NM, Turner AJ (2006) The involvement of lipid rafts in Alzheimer's disease. Mol Membr Biol 23:111-122

13. Dalakas MC (2006) Inflammatory, immune, and viral aspects of inclusion-body myositis. Neurology 66:S33-S38

14. Engel WK, Askanas V (2006) Inclusion-body myositis: clinical, diagnostic, and pathologic aspects. Neurology 66:S20-S29

15. Fratta P, Engel WK, McFerrin J, Davies KJ, Lin SW, Askanas V (2005) Proteasome inhibition and aggresome formation in sporadic inclusion-body myositis and in amyloid-beta precursor protein-overexpressing cultured human muscle fibers. Am J Pathol 167:517-526

16. Fukuchi K, Pham D, Hart M, Li L, Lindsey JR (1998) Amyloidbeta deposition in skeletal muscle of transgenic mice: possible model of inclusion body myopathy. Am J Pathol 153:1687-1693

17. Gil V, Nicolas O, Mingorance A, Urena JM, Tang BL, Hirata T, Saez-Valero J, Ferrer I, Soriano E, del Rio JA (2006) Nogo-A expression in the human hippocampus in normal aging and in Alzheimer disease. J Neuropathol Exp Neurol 65:433-444

18. He W, Lu Y, Qahwash I, Hu XY, Chang A, Yan R (2004) Reticulon family members modulate BACE1 activity and amyloid-beta peptide generation. Nat Med 10:959-965

19. He W, Hu X, Shi Q, Zhou X, Lu Y, Fisher C, Yan R (2006) Mapping of interaction mediating binding between BACE1 and RTN/ Nogo proteins. J Mol Biol 363:625-634

20. Jin LW, Hearn MG, Ogburn CE, Dang N, Nochlin D, Ladiges WC, Martin GM (1998) Transgenic mice over-expressing the C-99 fragment of betaPP with an alpha-secretase site mutation develop a myopathy similar to human inclusion body myositis. Am J Pathol 153:1679-1686

21. Kitazawa M, Green KN, Caccamo A, LaFerla FM (2006) Genetically augmenting Abeta42 levels in skeletal muscle exacerbates inclusion body myositis-like pathology and motor deficits in transgenic mice. Am J Pathol 168:1986-1997

22. Lee AS (2005) The ER chaperone and signaling regulator GRP78/ $\mathrm{BiP}$ as a monitor of endoplasmic reticulum stress. Methods 35:373-381

23. Marlow L, Cain M, Pappolla MA, Sambamurti K (2003) Beta-secretase processing of the Alzheimer's amyloid protein precursor (APP). J Mol Neurosci 20:233-239

24. McFerrin J, Engel WK, Askanas V (1998) Impaired innervation of cultured human muscle overexpressing Amyloid-betaPP experimentally and genetically: relevance to inclusion-body myopathies. Neuroreport 9:3201-3205

25. Ng CE, Tang BL (2002) Nogos and the Nogo-66 receptor: factors inhibiting CNS neuron regeneration. J Neurosci Res 67:559-565

26. Nogalska A, Engel WK, McFerrin J, Kokame K, Komano H, Askanas V (2006) Homocysteine-induced endoplasmic reticulum protein (Herp) is up-regulated in sporadic inclusion-body myositis and in endoplasmic reticulum stress-induced cultured human muscle fibers. J Neurochem 96:1491-1499

27. Nogalska A, Wojcik S, King Engel W, McFerrin J, Askanas V (2007) Endoplasmic reticulum stress induces myostatin precursor protein and NF-kappaB in cultured human muscle fibers: Relevance to inclusion body myositis. Exp Neurol 204:610-618

28. Sarkozi E, Askanas V, Johnson SA, Engel WK, Alvarez RB (1993) beta-Amyloid precursor protein mRNA is increased in inclusion-body myositis muscle. Neuroreport 4:815-818

29. Sarkozi E, Askanas V, Johnson SA, McFerrin J, Engel WK (1994) Expression of beta-amyloid precursor protein gene is developmentally regulated in human muscle fibers in vivo and in vitro. Exp Neurol 128:27-33

30. Satoh J, Onoue H, Arima K, Yamamura T (2005) Nogo-A and nogo receptor expression in demyelinating lesions of multiple sclerosis. J Neuropathol Exp Neurol 64:129-138

31. Sugarman MC, Yamasaki TR, Oddo S, Echegoyen JC, Murphy MP, Golde TE, Jannatipour M, Leissring MA, LaFerla FM (2002) Inclusion body myositis-like phenotype induced by transgenic overexpression of beta APP in skeletal muscle. Proc Natl Acad Sci USA 99:6334-6339

32. Teng FY, Ling BM, Tang BL (2004) Inter- and intracellular interactions of Nogo: new findings and hypothesis. J Neurochem 89:801-806

33. Tun H, Marlow L, Pinnix I, Kinsey R, Sambamurti K (2002) Lipid rafts play an important role in A-beta biogenesis by regulating the beta-secretase pathway. J Mol Neurosci 19:31-35

34. Vattemi G, Engel WK, McFerrin J, Buxbaum JD, Pastorino L, Askanas V (2001) Presence of BACE1 and BACE2 in muscle fibres of patients with sporadic inclusion-body myositis. Lancet 358:1962-1964

35. Vattemi G, Kefi M, Engel WK, Askanas V (2003) Nicastrin, a novel protein participating in amyloid-beta production, is overexpressed in sporadic inclusion-body myositis muscle. Neurology 60:A315

36. Vattemi G, Engel WK, McFerrin J, Pastorino L, Buxbaum JD, Askanas V (2003) BACE1 and BACE2 in pathologic and normal human muscle. Exp Neurol 179:150-158

37. Vattemi G, Engel WK, McFerrin J, Askanas V (2004) Endoplasmic reticulum stress and unfolded protein response in inclusion body myositis muscle. Am J Pathol 164:1-7

38. Vetrivel KS, Thinakaran G (2006) Amyloidogenic processing of beta-amyloid precursor protein in intracellular compartments. Neurology 66:S69-73 
39. Wang X, Chun SJ, Treloar H, Vartanian T, Greer CA, Strittmatter SM (2002) Localization of Nogo-A and Nogo-66 receptor proteins at sites of axon-myelin and synaptic contact. J Neurosci 22:5505-5515

40. Wojcik S, Engel WK, McFerrin J, Askanas V (2005) Myostatin is increased and complexes with amyloid-beta within sporadic inclusionbody myositis muscle fibers. Acta Neuropathol (Berl) 110:173-177

41. Wojcik S, Engel WK, McFerrin J, Paciello O, Askanas V (2006) AbetaPP-overexpression and proteasome inhibition increase alphaB-crystallin in cultured human muscle: relevance to inclusionbody myositis. Neuromuscul Disord 16:839-844
42. Wojcik S, Engel WK, Askanas V (2006) Increased expression of Nogo-A in ALS muscle biopsies is not unique for this disease. Acta Myologica 25:116-118

43. Wojcik S, Nogalska A, McFerrin J, Engel WK, Oledzka G, Askanas V (2007) Myostatin precursor protein is increased and associates with amyloid- $\beta$ precursor protein in inclusion-body myositis culture model. Neuropathol Appl Neurobiol 33:238-242

44. Yan R, Shi Q, Hu X, Zhou X (2006) Reticulon proteins: emerging players in neurodegenerative diseases. Cell Mol Life Sci 63:877889 